\title{
Presentación \\ La vivienda tras la Crisis Financiera Global: nueva visión, viejos problemas
}

\author{
Paloma Taltavull de La Paz \\ Universidad de Alicante
}

El monográfico que se presenta en este número, como su título indica, está dedicado al mercado de la vivienda. Si hay algún análisis económico referido a un sector que haya cambiado radicalmente en la última década, ese ha sido el de este mercado. Tradicionalmente, este sector ha sido estudiado desde la óptica de los mercados locales y de la economía urbana con gran acento microeconómico, de forma que cualquier enfoque que se refiriese a un efecto contagio o de interacción con otros sectores (salvo el de la pobreza), era considerado fuera de lugar. Aunque muy avanzados y rigurosos, los trabajos que profundizaban en el conocimiento de los mercados residenciales no solían traspasar la frontera de las áreas urbanas o del análisis del propio mercado, $\mathrm{y}$, mucho menos, saltar a los ámbitos macroeconómicos con una relevancia similar a la de cualquier otro indicador agregado.

Desde la Crisis Financiera Global (CFG), y asociados a la evolución de los mercados financieros y al colapso de los mercados de derivados, las implicaciones de un desajuste en los mercados de viviendas han sido reconocidas como elemento fundamental que pueden contribuir a (y ser aviso de) los desequilibrios de una economía. Así lo ha entendido la Unión Europea cuando construyó el panel de indicadores de desequilibrio en su Procedimiento de Déficit Excesivo (MIP y EIP), al incluir como indicador básico (el número 6) el crecimiento de los precios de las viviendas. Este reconocimiento seguía al flujo de trabajos y debates que, a partir del estallido de la CFG, han ido analizando el papel de la evolución de los precios residenciales, su implicación en la responsabilidad de la propia crisis y de la larga recesión posterior e, incluso, la creciente evidencia sobre la relación entre los ciclos inmobiliarios a nivel global que reflejan un fuerte efecto de transmisión entre los mercados de viviendas en el mundo.

Por lo reciente de los análisis y de los hechos, todavía existe un fuerte debate sobre las interacciones del mercado de la vivienda con el resto de la economía. Es ampliamente aceptado el intenso efecto motor de la construcción de viviendas (oferta) sobre el contexto económico, aunque se habían olvidado importantes trabajos que defendían que una economía desarrollada no crece de forma apropiada sin un mercado residencial asentado y fuerte. Por otro lado, hay también acuerdo sobre la relevancia social de la vivienda como elemento que cubre una necesidad básica, pero tras más de una década disfrutando de un mercado residencial con ausencia de los 
problemas derivados de la falta de accesibilidad a las viviendas, también se habían olvidado situaciones de escasez y de la necesidad de que la política económica resolviese el fallo de mercado que se manifiesta en la falta de provisión residencial para los hogares más desfavorecidos.

Por último, un nuevo grupo de interacciones se han puesto de manifiesto de la forma más cruda con las dos crisis sucesivas tras 2007 relacionados con súbitos cambios en los precios de las viviendas, básicamente en su efecto sobre los valores de las carteras de inversión, y en el equilibrio (y riesgo) relacionado con la exposición de los sistemas financieros a los créditos hipotecarios. Los trabajos más modernos identifican la relación de los precios residenciales con los mecanismos de transmisión de la política monetaria, de manera que los cambios en los precios de las viviendas (derivados de nuevas condiciones en sus mercados) pueden llegar a tener efectos multiplicadores a través del canal del precio de los activos, afectando a los objetivos fijados por el regulador monetario. En este último apartado, importantes centros de investigación asociados a los bancos centrales están realizando contribuciones que permiten comprender cómo se pueden transmitir los cambios en los valores de las viviendas a la economía en su conjunto y a otras economías relacionadas.

En resumen, problemas antiguos y (quizás) nuevos asociados a un mercado clave en cualquier economía que han vuelto a colocarse en primera línea de la investigación buscando explicaciones para la evolución económica en su conjunto. En este caso, se requiere de su análisis y monitorización continuada dado que este mercado, el de la vivienda, posee un marcado equilibrio inestable por su propia naturaleza.

En España, el análisis del mercado residencial no ha recibido demasiada atención a pesar de su relevancia dentro de la estructura económica. Si bien otras economías también lo tienen, en la española el sector residencial cubre ampliamente dos objetivos: las necesidades básicas de la población, y la de residencia asociada a un volumen nada despreciable también de migrantes temporales. Estos últimos han sido analizados dentro de otro sector también clave para la economía española, como es el turismo, desviando la atención del sector residencial como proveedor clave de servicios (de residencia).

Sin lugar a dudas, el crecimiento del sector de la vivienda ha seguido las pautas del primero de estos dos objetivos, la demanda básica. España ha experimentado un aumento continuado de la demanda demográfica durante varias décadas (primero por la migración interna de los sesenta y setenta, después por la llegada de los babyboomers en los ochenta y noventa, y, a continuación, por la oleada de inmigración en la primera década del siglo XXI), generando una presión extraordinaria sobre los mercados residenciales que se ha resuelto con una intensa respuesta de la oferta.

Esta situación sostenida ha generado la percepción de que, en España, la oferta de viviendas crece de forma continuadae intensa como status quo, aunque, tras la comparación con lo ocurrido en otras economías, la idea de que el elemento diferencial español es la flexibilidad de la oferta, toma fuerza. Existe evidencia que permite comprender las razones de por qué la oferta ha reaccionado de forma tan intensa en España durante décadas, y apuntan al rol que han tenido, sucesivamente, la política de vivienda, primero, 
y la inversión privada, después, en el intenso proceso de edificación experimentado en los últimos cincuenta años, que ha generado dos grandes ciclos constructores (de 15 años cada uno) y multiplicado casi por tres el stock residencial entre 1960 y 2011. El proceso no ha estado exento de crisis relevantes que han afectado de distintas formas al sistema financiero, no solo la reciente y conocida, desde 2008 tras la CFG, sino otra anterior localizada a finales de la década de los setenta del siglo Xx.

Quitando estos sucesos, lo cierto es que el stock ha crecido a una tasa lo suficientemente importante como para dar cobijo a la población, que también lo hacía, y dar lugar, como resultado, a una situación que por invisible pasa desapercibida, como es que la mayoría de los hogares españoles (en cualquier nivel de renta) disponen de una vivienda. Este proceso ha alejado del ámbito español las sombras de grandes áreas de infravivienda que se generaron en otros núcleos urbanos como respuesta a similares presiones demográficas a las experimentadas en España, esquivando la existencia de áreas de concentración de pobreza difíciles de revertir.

Con las nuevas estructuras institucionales, y la CFG, las necesidades y los desajustes en el mercado de la vivienda han surgido de nuevo. La severidad de la crisis económica ha aflorado los problemas más graves que se pueden generar este mercado, como es la pobreza inducida por la falta de vivienda, con el aumento de los desahucios y de las necesidades residenciales.

La situación actual de la economía española, por tanto, requiere de una revisión de la situación en la que se encuentra el mercado de la vivienda. El objetivo de este monográfico es la evaluación de las cuestiones más relevantes que emergen tras la crisis que están relacionadas con la vivienda y los efectos del colapso de largo periodo expansivo y de la crisis económica y financiera resultante. Se ha dividido el monográfico en tres bloques. El primero aborda la situación del mercado de la vivienda desde un punto de vista estrictamente económico, con análisis desde la oferta y la demanda. El segundo trata ampliamente distintos aspectos de la política de vivienda, y el tercero, cuestiones relacionadas con el sistema financiero. Este último es más reducido, no por menos importante, sino porque el estado de las investigaciones actuales no ha alcanzado aún su cénit.

Dentro del primer bloque, los dos primeros artículos enmarcan la situación del mercado y de sus efectos. Julio Rodríguez da una visión integrada de la situación reciente del mercado de la vivienda en España asociada a los cambios en el ciclo económico y sus vinculaciones con otros sectores. Es un planteamiento que integra la mayor parte de las causas y las consecuencias de la evolución de los mercados con la vivienda y da una idea nítida de la complejidad en el esquema de relaciones que vincula este sector con el conjunto de la economía. Concluye que la caída en el sector (de oferta) se ha estabilizado y da algunos consejos sobre medidas de política económica a seguir.

Michael White y Paloma Taltavull aportan una revisión de la literatura que esquematiza las relaciones del sector de la vivienda y la economía en su conjunto. Organizan las aportaciones de manera que muestran los mecanismos que interrelacionan el mercado de vivienda con los hechos socioeconómicos. Por ejemplo, 
muestran el círculo virtuoso de crecimiento económico generado por la demanda de viviendas en propiedad; las relaciones entre los cambios de la demanda de viviendas con el efecto diferencial de los precios residenciales y sus implicaciones y definen la relación entre la política monetaria (o los cambios en variables monetarias) con el mercado de viviendas, en este último caso, profundizando en la literatura que identifica el «acelerador residencial».

Javier Barrios aborda uno de los aspectos cruciales de este mercado como es la relación de la tenencia de vivienda en propiedad y el empleo. Tradicionalmente se ha defendido que la tenencia en propiedad reduce la movilidad del trabajo y contribuye a mayores tasas de desempleo. El resultado de su análisis es contundente sobre la falta de relación entre ambos aspectos, disgregando la forma de tenencia con la probabilidad de encontrar un empleo. Concluye, aportando evidencia empírica, que los hogares con tenencia en propiedad tienen mayores probabilidades de tener empleo que en los hogares en alquiler.

El análisis sobre la oferta de vivienda nueva se realiza en el cuarto artículo. Paloma Taltavull, continuando análisis previos sobre la respuesta de los sectores promotores a los impulsos de mercado, estima elevadas elasticidades de oferta de viviendas nuevas para la mayor parte de las regiones españolas en presencia de la disponibilidad de suelo. Su modelo analiza el periodo 1990-2015, con lo que tiene la oportunidad de aproximar el efecto de la crisis financiera sobre la oferta de vivienda nueva. Sus resultados sobre las elevadas elasticidades contribuyen a explicar por qué se ha producido un ajuste severo en la edificación (más que en los precios) en la mayor parte de las regiones, y cómo solo seis de las quince evaluadas muestran una sensibilidad estadísticamente significativa de la edificación de vivienda nueva ante la oferta de suelo.

El bloque II se dedica íntegramente a distintos aspectos relacionados con la política de vivienda. Dos primeros trabajos introducen la necesidad de esta política en una sociedad desarrollada. En el primero, Francisco Juárez plantea el concepto de pobreza en vivienda y accesibilidad y la fórmula en la que se calcula en la literatura. La pobreza en vivienda es un fenómeno poco analizado pero es determinante de la calidad de vida de los hogares y puede abocarles a caer por debajo de la línea de pobreza. El autor analiza si los hogares con tenencia en propiedad tienen mayores niveles de pobreza en vivienda que los que ostentan otras fórmulas de tenencia, en un intento por identificar si la elevada tenencia en propiedad protege de la pobreza a los hogares españoles. Mediante la definición y estimación de un índice combinado de accesibilidad, observa la proporción de hogares para los que la pobreza en vivienda les hace caer por debajo del umbral de pobreza, es decir, el aumento de la pobreza derivado de la dependencia del pago de la vivienda. Sus conclusiones ponen de manifiesto la existencia de este fenómeno no observado para España que afecta a un 11,4 por 100 del total de los hogares pobres. El trabajo también concluye que la tasa de propiedad ha protegido de la pobreza a una parte de la población y que los problemas de accesibilidad afectan en mayor medida a los hogares alquilados, lo que es un incentivo (precaución) para tratar de acceder a la propiedad. 
Kenneth Gibb plantea las situaciones de falta de vivienda en el Reino Unido tras la crisis financiera, y cómo el fallo de provisión desde el mercado (que habría actuado favorablemente hasta 2007) no ha sido compensado con la política económica. El impacto de la crisis, con reducción drástica de construcción y precios de suelo, no se vio compensado con caídas sustanciales de los precios residenciales lo que exacerbó los problemas de accesibilidad. Describe cómo las políticas de austeridad de los distintos gobiernos junto con la reducción del crédito tras la CFG, generaron problemas de accesibilidad severos y una falta de vivienda accesible, sin solución hasta el momento.

Tras el planteamiento de las situaciones de pobreza y desajuste social relacionados

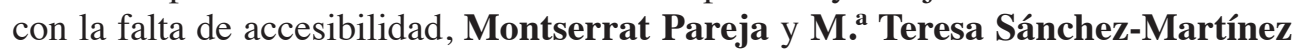
explican el sistema de política de viviendas en España y su situación tras la crisis financiera. Tras describir los elementos fundamentales de la política de vivienda existente, reclaman medidas más activas, cambios en la regulación sobre el alquiler y el desarrollo de un parque de viviendas sociales para cubrir las eventuales necesidades residenciales que han crecido tras la $\mathrm{CFG}$.

La similitud en la contracción de la edificación pública con la que ha ocurrido en la privada tras la crisis plantea la duda de si la política de edificación de vivienda pública es autónoma o está vinculada al ciclo de edificación privado, en España. Montserrat Díaz, Mar Llorente y Paloma Taltavull se plantean esta cuestión y calculan si existen efectos desplazamiento (crowding-in o -out) entre ambos ciclos público y privado evaluando, así, si las decisiones finales de construcción de vivienda pública se encuentran ancladas a las decisiones de los sectores privados. Diseñando un modelo por provincias, las autoras demuestran que las relaciones son asimétricas y diferentes entre las regiones analizadas, consistentes con su distinta tipología de mercados y con las regulaciones sobre vivienda pública dispares. En concreto, encuentran que hay efectos expulsión desde la vivienda pública a la privada en Cáceres y Madrid; el efecto contrario en Vizcaya; ciclos independientes en Guadalajara y Zaragoza; y una provincia, Alicante, donde ambos ciclos interaccionan bidireccionalmente. Las autoras concluyen que la forma de aplicar la política de vivienda debe tener en cuenta estas relaciones dispares entre regiones.

El último trabajo en este bloque evalúa los aspectos fiscales de la política de vivienda. Miguel Ángel López se plantea los efectos de la política impositiva sobre el stock residencial y sobre los incentivos de invertir en vivienda. Tras años en que el ordenamiento fiscal se ha sumado a una política de vivienda activa incentivadora de la inversión, los cambios del tratamiento fiscal y la desaparición de las desgravaciones por compra de vivienda que se producen a partir de la CFG, han generado una situación de incentivo negativo al ahorro y a la inversión, contribuyendo a la caída en la demanda de vivienda que se experimenta en España y a un efecto perverso de transferencia de renta desde los segmentos jóvenes y cohortes futuras, a los de mayor edad. En sus comentarios finales, el autor aboga por la bondad de los incentivos a la inversión en vivienda para revertir ese círculo vicioso. Este instrumento, abandonado por la política fiscal en España, es posiblemente el único que pueda conseguir una redistribución en favor de los jóvenes en estos momentos. 
El bloque III se dedica al papel del sistema financiero. Dados los enormes cambios que se han producido simultáneamente tras la CFG en el sistema financiero español (afectando al mercado hipotecario), en la regulación europea (con las normas de Basilea II y III), y en la situación financiera internacional, abordar apropiadamente el papel de la financiación y su rol hubiese llevado un número especial por sí mismo. La relevancia de este sector queda patente con la estructura de funcionamiento explicada en el primer bloque temático, por lo que en este número especial se ha optado por abordar los cambios en la regulación que afectan al sistema hipotecario y al crédito a la vivienda.

Lorena Mullor e Irene Peña explican las directivas y reglamentos de capital y liquidez a los que tienen que someterse las instituciones financieras en España y que determinan la concesión de créditos hipotecarios. Concluyen que la nueva regulación no debería tener un impacto excesivo sobre el mercado hipotecario español, aunque manifiestan preocupación por el potencial endurecimiento de los criterios aplicados al crédito hipotecario, el aumento de los requerimientos de liquidez, y la mayor información requerida en la concesión de créditos, entre otros requisitos, que pueden llevar a una burocratización de los procesos de concesión del crédito hipotecario que penalizará la recuperación de los flujos normales.

Muchos aspectos han quedado por abordar sobre la vivienda y dejan abierto un ámbito amplio para ulteriores investigaciones. Muchas de ellas están en la arena financiera, como el impacto de la reestructuración financiera sobre la estructura y funcionamiento del mercado hipotecario español, las fórmulas de financiación de la inversión inmobiliaria con el papel de las Socimis y otra tipología de activos, el proceso de ajuste a través del Sareb o el papel de la inversión extranjera, entre otros. Hay también numerosos aspectos que afectan a la demanda que se desconocen en España, como son el papel de la inmigración temporal, las fórmulas de tenencia y su rol social, el comportamiento y formación de los precios. Hay numerosas cuestiones desde la oferta que tienen aún que ser analizadas, como la realidad de las viviendas vacías o el papel de la industria de la construcción en la determinación de la oferta, y, de gran relevancia, como la adaptación del stock a los requisitos de reducción en el consumo energético, entre otros. Temas importantes que, espero, generen curiosidad entre los investigadores a partir de este monográfico. 